\title{
THE CALIFORNIA PLANET SURVEY. III. A POSSIBLE 2:1 RESONANCE IN THE EXOPLANETARY TRIPLE SYSTEM HD 37124*
}

\author{
J. T. Wright ${ }^{1,2}$, Dimitri Veras ${ }^{3}$, Eric B. Ford ${ }^{3}$, John Asher Johnson ${ }^{4}$, G. W. Marcy ${ }^{5}$, A. W. Howard ${ }^{5,6,9}$,

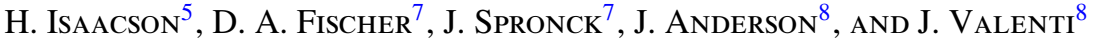 \\ ${ }^{1}$ Department of Astronomy, 525 Davey Lab, The Pennsylvania State University, University Park, PA 16802, USA \\ ${ }^{2}$ Center for Exoplanets and Habitable Worlds, The Pennsylvania State University, University Park, PA 16802, USA \\ ${ }^{3}$ Department of Astronomy, University of Florida, 211 Bryant Space Science Center, P.O. Box 112055, Gainesville, FL 32611-2055, USA \\ ${ }^{4}$ Department of Astronomy, California Institute of Technology, MC 249-17, Pasadena, CA, USA \\ ${ }^{5}$ Department of Astronomy, 601 Campbell Hall, University of California, Berkeley, CA 94720-3411, USA \\ ${ }^{6}$ Space Sciences Laboratory, University of California, Berkeley, CA, USA \\ ${ }^{7}$ Department of Astronomy, Yale University, New Haven, CT 06511, USA \\ ${ }^{8}$ Space Telescope Science Institute, 3700 San Martin Drive, Baltimore, MD 21218, USA \\ Received 2010 August 22; accepted 2011 January 5; published 2011 March 9
}

\begin{abstract}
We present new radial velocities from Keck Observatory and both Newtonian and Keplerian solutions for the triple-planet system orbiting HD 37124. The orbital solution for this system has improved dramatically since the third planet was first reported in Vogt et al. with an ambiguous orbital period. The period ambiguity is resolved, and the outer two planets have an apparent period commensurability of 2:1. A dynamical analysis finds both resonant and non-resonant configurations consistent with the radial velocity data and constrains the mutual inclinations of the planets to be $<\sim 30^{\circ}$. We discuss HD 37124 in the context of the other 19 exoplanetary systems with apparent period commensurabilities, which we summarize in a table. We show that roughly one in three well-characterized multiplanet systems has a apparent low-order period commensurability, which is more than would naïvely be expected if the periods of exoplanets in known multiplanet systems were drawn randomly from the observed distribution of planetary orbital periods.
\end{abstract}

Key words: planetary systems - stars: individual (HD 37124)

\section{INTRODUCTION}

To date, over 50 exoplanetary systems with more than one planet have been discovered, including the extraordinary detections of the first exoplanets orbiting the pulsar PSR B1257+12 (Wolszczan \& Frail 1992; Wolszczan 1994); the imaged system orbiting HR 8799; those discovered during the microlensing event OGLE-2006-BLG-109L (Gaudi et al. 2008); several systems discovered by transit, including four or five ${ }^{10}$ multiply transiting systems from the Kepler mission (Steffen et al. 2010); and 43 systems discovered by radial velocity (RV) searches (Wright 2010). The RV systems include the four-planet systems $\mu$ Ara (Santos et al. 2004; Pepe et al. 2007), GJ 581 (Mayor et al. 2009), and GJ 876 (Rivera et al. 2005, 2010) and the five-planet system orbiting 55 Cancri (Fischer et al. 2008). Of all these multiplanet systems, only four are known to host three or more giant ${ }^{11}$ planets with well-determined orbital parameters: $v$ And (Butler et al. 1999), HIP 14810 (Wright et al. 2009b), $\mu$ Ara (Pepe et al. 2007), and HD 37124 (Vogt et al. 2005).

HD 37124 (HIP 26381) is a $0.85 M_{\odot}$ metal-poor $([\mathrm{Fe} / \mathrm{H}]=$ -0.44; Valenti \& Fischer 2005) G4 dwarf ( $V=7.7)$. Vogt et al. (2000) announced a Jovian, $P \sim 150$ days, planet orbiting HD 37124 from HIRES data taken at Keck Observatory as part of the California and Carnegie Planet Search. Further monitoring of the star revealed substantial long-term residuals. Butler et al.

\footnotetext{
* Based on observations obtained at the W. M. Keck Observatory, which is operated jointly by the University of California and the California Institute of Technology. The Keck Observatory was made possible by the generous financial support of the W. M. Keck Foundation.

9 Townes Postdoctoral Fellow.

10 KOI 877 may be a blend of two, separately transiting systems.

${ }_{11} M \sin i>0.2 M_{\text {Jup }}$.
}

(2003) fit these residuals with an eccentric, 1940 day planet, but noted that the solution was not unique (and Goździewski 2003 showed that this fit was, in fact, unstable).

After collecting two more years of data, Vogt et al. (2005) were able to report the detection of a third planet in the system, though with an ambiguity, while the $b$ and $c$ components had clearly defined periods, the $d$ component could be fit nearly equally well with periods of either 2300 days or 29.32 days, the latter likely being an alias due to the lunar cycle. ${ }^{12}$ Wright (2010) reported that recent Keck velocities had resolved the ambiguity qualitatively in favor of the longer orbital period. Goździewski et al. (2006) explored the many possible dynamical configurations consistent with the Vogt et al. (2005) velocities, including many resonant solutions. Goździewski et al. (2008) used the system to demonstrate a fast MENGO algorithm, but they did not explore the 2:1 resonance, as the data did not seem to favor it at the time.

We present new Keck observations, and these data provide for a unique orbital solution for the outer planet. The outer planet period we find is more consistent with the original period reported by Butler et al. (2003) than the refined orbit of Vogt et al. $(2005)^{13}$ (though we find a much lower eccentricity). Herein, we present the entire history of Keck velocities obtained for this star and present self-consistent orbital solutions showing that the outer two planets are in or very near a 2:1 mean-motion

\footnotetext{
12 Time on the Keck telescopes dedicated to observing bright, planet search targets with HIRES is usually assigned during bright or gray time; the resulting scarcity of data points during new moon can interact with planetary signals to create spurious, aliased solutions.

13 Vogt et al. (2005) opted to refer to the new, 840 day signal as the $c$ component, despite the prior 1940 day fit of Butler et al. (2003), because that prior fit was so speculative, and because their new fit put the very existence of a 1940 day periodicity in some doubt.
} 
resonance (MMR). This is the twentieth exoplanetary system to be near an MMR and only the tenth system with an apparent 2:1 commensurability.

Period commensurabilities (PCs) represent important dynamical indicators in the solar system and have been linked with observables and formation mechanisms (Goldreich 1965). The near-5:2 PC of Jupiter and Saturn, also known as "The Great Inequality," might be the remnant of a divergent resonant crossing that produced the current architecture of the outer solar system, the Late Heavy Bombardment, and the Trojan Asteroids (Gomes et al. 2005; Morbidelli et al. 2005; Tsiganis et al. 2005a, 2005b). The populations of the asteroid belt and the Kuiper Belt, exemplified by the PC and near-PC-populated Kirkwood Gaps (e.g., Tsiganis et al. 2002) the Plutinos (3:2 PCs with Pluto and Neptune) and the twotinos (2:1 PCs with Pluto and Neptune; e.g., Murray-Clay \& Chiang 2005; Chiang \& Jordan 2002), have implications for the migratory history of Jupiter and Neptune and the prospect of, e.g., secular resonant sweeping (e.g., Nagasawa et al. 2005). Near PCs found in satellite and ring systems have had direct observational consequences; the Saturnian satellite Pandora was $\sim 19^{\circ}$ behind its predicted orbital longitude in a 1995 ring plane crossing (French et al. 2003) due to its 121:118 PC with neighboring satellite Prometheus.

By extension, we may anticipate similar importance in the growing number of exoplanetary systems exhibiting PCs. In extrasolar systems, MMRs have been interpreted as the indication of convergent migration in multiplanet systems (e.g., Thommes \& Lissauer 2003; Kley et al. 2004; Papaloizou \& Szuszkiewicz 2005). Several subsequent studies (Beaugé et al. 2006; Terquem \& Papaloizou 2007; Pierens \& Nelson 2008; Podlewska \& Szuszkiewicz 2008, 2009; Libert \& Tsiganis 2009; Rein \& Papaloizou 2009; Papaloizou \& Terquem 2010; Rein et al. 2010; Zhang \& Zhou 2010a, 2010b) exploring convergent migration for a variety of masses, separations, and disk properties have found many regions of mass and orbital element phase space in which planets are easily captured through this mechanism.

\section{VELOCITIES AND ORBITAL SOLUTION}

Table 1 contains RV measurements for HD 37124 from the HIRES spectrograph (Vogt et al. 1994) at Keck Observatory obtained by the California Planet Survey consortium using the iodine technique (Butler et al. 1996). Note that the quoted errors are our internal (random) errors, with no "jitter" included (Wright 2005).

These velocities supersede our previously published velocities for this star, as we continue to refine our data reduction pipeline. Our ever-evolving RV pipeline is descended in spirit and form from that described in Butler et al. (1996), but includes many small and large technical improvements, a thorough discussion of which is beyond the scope of this manuscript. Some details can be found in Section 4.1 of Howard et al. (2010), Section 3 of Howard et al. (2009), and in Batalha et al. (2011).

One issue of instant relevance is that in 2004 August the HIRES CCD detector was upgraded to a CCD mosaic. The old Tektronix 2048 EB2 engineering-grade CCD displayed a variable instrumental profile asymmetry due to a charge transfer inefficiency which manifested itself as small changes in a star's measured RV as a function of exposure time (i.e., raw counts on the chip.) We apply an empirical, spectral-type dependent model based to correct this effect for velocities measured prior to the detector change. The new CCD mosaic shows no evidence of this effect, but as a consequence of the switch there is a small
Table 1

Radial Velocities for HD 37124

\begin{tabular}{|c|c|c|c|}
\hline $\begin{array}{c}\text { Time } \\
\text { JD-2440000 }\end{array}$ & $\begin{array}{l}\text { Velocity } \\
\left(\mathrm{m} \mathrm{s}^{-1}\right)\end{array}$ & $\begin{array}{c}\text { Uncertainty } \\
\left(\mathrm{m} \mathrm{s}^{-1}\right)\end{array}$ & $\mathrm{CCD}$ \\
\hline 10420.04655 & 54.81 & 1.5 & 1 \\
\hline 10546.73646 & 28.74 & 1.2 & 1 \\
\hline 10837.76625 & 6.32 & 1.6 & 1 \\
\hline 10838.94868 & 6.38 & 1.7 & 1 \\
\hline 10861.80464 & 17.89 & 1.4 & 1 \\
\hline 11069.03617 & -3.99 & 1.4 & 1 \\
\hline 11070.13190 & -1.66 & 1.3 & 1 \\
\hline 11071.11494 & 1.28 & 1.6 & 1 \\
\hline 11072.12947 & -11.58 & 1.5 & 1 \\
\hline 11073.02962 & -8.72 & 1.3 & 1 \\
\hline 11172.89571 & 39.40 & 1.6 & 1 \\
\hline 11226.78065 & -0.48 & 1.4 & 1 \\
\hline 11227.78167 & -2.05 & 1.4 & 1 \\
\hline 11228.74293 & -11.01 & 1.3 & 1 \\
\hline 11412.14161 & -33.76 & 1.6 & 1 \\
\hline 11543.98278 & -31.36 & 1.4 & 1 \\
\hline 11550.94262 & -44.57 & 1.4 & 1 \\
\hline 11551.94008 & -46.20 & 1.5 & 1 \\
\hline 11552.89162 & -47.97 & 1.5 & 1 \\
\hline 11580.76121 & -36.28 & 1.8 & 1 \\
\hline 11581.83559 & -36.17 & 1.4 & 1 \\
\hline 11582.78849 & -37.36 & 1.4 & 1 \\
\hline 11583.72387 & -35.69 & 1.5 & 1 \\
\hline 11884.04436 & -14.73 & 1.6 & 1 \\
\hline 11900.03518 & 3.01 & 1.4 & 1 \\
\hline 11974.80019 & 39.56 & 1.4 & 1 \\
\hline 12007.74522 & 4.48 & 1.5 & 1 \\
\hline 12242.99064 & 48.20 & 1.5 & 1 \\
\hline 12333.94545 & -19.13 & 1.7 & 1 \\
\hline 12334.78556 & -12.82 & 1.7 & 1 \\
\hline 12536.12848 & 10.64 & 1.8 & 1 \\
\hline 12537.08597 & 10.28 & 1.7 & 1 \\
\hline 12573.03767 & 27.38 & 1.6 & 1 \\
\hline 12574.99934 & 27.48 & 1.7 & 1 \\
\hline 12576.02212 & 23.58 & 1.6 & 1 \\
\hline 12600.99996 & 8.06 & 1.7 & 1 \\
\hline 12602.03213 & 7.02 & 1.7 & 1 \\
\hline 12925.01639 & 13.03 & 1.7 & 1 \\
\hline 13044.77359 & 36.56 & 1.6 & 1 \\
\hline 13045.74638 & 32.78 & 1.5 & 1 \\
\hline 13072.85948 & -2.85 & 1.7 & 1 \\
\hline 13240.13983 & -34.52 & 1.5 & 2 \\
\hline 13302.13193 & -4.28 & 1.6 & 2 \\
\hline 13302.97959 & -5.69 & 1.4 & 2 \\
\hline 13338.96446 & 15.77 & 1.2 & 2 \\
\hline 13340.09520 & 16.45 & 1.5 & 2 \\
\hline 13368.88930 & -6.51 & 1.0 & 2 \\
\hline 13369.78156 & -6.60 & 1.0 & 2 \\
\hline 13425.87197 & -39.98 & 1.4 & 2 \\
\hline 13426.82980 & -39.12 & 1.3 & 2 \\
\hline 13428.77030 & -36.85 & 1.3 & 2 \\
\hline 13483.72749 & 19.49 & 1.0 & 2 \\
\hline 13723.90630 & -7.28 & 1.6 & 2 \\
\hline 13841.76427 & 34.09 & 1.4 & 2 \\
\hline 14544.83023 & 25.40 & 1.6 & 2 \\
\hline 14545.78169 & 26.99 & 1.4 & 2 \\
\hline 14546.78977 & 24.28 & 1.3 & 2 \\
\hline 14718.08322 & 43.60 & 1.6 & 2 \\
\hline 14806.91704 & -7.74 & 1.5 & 2 \\
\hline 14810.89031 & -9.50 & 1.7 & 2 \\
\hline 14838.94681 & 0.39 & 1.8 & 2 \\
\hline 14864.95362 & 29.45 & 1.8 & 2 \\
\hline 14929.76349 & -5.49 & 1.7 & 2 \\
\hline 15135.00085 & -21.29 & 1.6 & 2 \\
\hline 15172.99171 & 12.60 & 1.6 & 2 \\
\hline 15229.78574 & -12.32 & 1.6 & 2 \\
\hline
\end{tabular}


Table 2

Best-fit Kinematic Orbital Elements for Exoplanets in the HD 37124 System

\begin{tabular}{lccc}
\hline \hline \multicolumn{1}{c}{ Parameter } & $b$ & $c$ & $d$ \\
\hline$P$ (day) & $154.378 \pm 0.089$ & $885.5 \pm 5.1$ & $1862 \pm 38$ \\
$T_{p}(\mathrm{JD}-2440000)$ & $10305 \pm 11$ & $9534 \pm 11$ & $8558 \pm 11$ \\
$e$ & $0.054 \pm 0.028$ & $0.125 \pm 0.055$ & $0.16 \pm 0.14$ \\
$\omega(\mathrm{deg})$ & $130^{\mathrm{a}}$ & $53 \pm 17$ & $0^{\mathrm{a}}$ \\
$K\left(\mathrm{~m} \mathrm{~s}^{-1}\right)$ & $28.50 \pm 0.78$ & $15.4 \pm 1.2$ & $12.8 \pm 1.3$ \\
$M \sin i\left(M_{\text {Jup }}\right)$ & $0.675 \pm 0.017$ & $0.652 \pm 0.052$ & $0.696 \pm 0.059$ \\
$a(\mathrm{AU})$ & $0.53364 \pm 0.00020$ & $1.7100 \pm 0.0065$ & $2.807 \pm 0.038$ \\
\hline RMS & & 4.03 & \\
Jitter & & $4 \mathrm{~m} \mathrm{~s}^{-1}$ & \\
$\chi_{v}^{2}$ & & 0.8 & \\
$N_{\text {obs }}$ & 66 & \\
\hline
\end{tabular}

Notes. ${ }^{\text {a }}$ Orbit is consistent with circular, so errors in $\omega$ are large; see Butler et al. (2006) for a fuller explanation.

velocity offset between data sets that span the two detector sets similar to the detector-to-detector offsets discussed in Gregory $\&$ Fischer (2010). These offsets could, in principle, be different for every target.

Analysis of RV standards and known planetary systems show that such an offset is usually small-of order $5 \mathrm{~m} \mathrm{~s}^{-1}$-and very often consistent with zero. As a result, we report two independent data sets for this system in Table 1, one from each of the two detectors. We solve for the detector offset as an unconstrained free parameter. The times of observation are given in JD-2440000.

We fitted the data using the publicly available multiplanet RVfitting IDL package RV_FIT_MP, described in Wright \& Howard (2009). In Table 2, we present our three-planet Keplerian (kinematic) fit, ${ }^{14}$ which yields RMS residuals of $4.4 \mathrm{~m} \mathrm{~s}^{-1}$, and we plot the fit and velocities in Figure 1. We find a best-fit offset between CCDs of $4.8 \mathrm{~m} \mathrm{~s}^{-1}$. The orbital parameters and their uncertainties were determined from 100 bootstrapped trials (as described in Marcy et al. 2005; Butler et al. 2006; Wright et al. 2007). The orbital fits and dynamical analysis herein are put forth under the assumption that the velocities are not detectably influenced by additional, unmodeled planets in the system. We have integrated these orbital parameters for 10 Myr using the methods described in Section 3 assuming coplanarity and found them to yield a stable configuration.

The residuals to this fit have an RMS $4.03 \mathrm{~m} \mathrm{~s}^{-1}$ and show with no significant periodogram peak at any period. The tallest peak is at 3.81 days. We have run a Monte Carlo False Alarm Probability (FAP; e.g., Wright 2010) analysis on these residuals of our best fit for this tallest peak and find similarly good fits in $50 \%$ of velocity-scrambled trials, consistent with noise. We thus conclude that our model is sufficient to explain the data and that there are no other statistically significant planetary signals detected.

\section{NEWTONIAN FITS AND STABILITY ANALYSIS}

\subsection{MCMC Analyses}

We studied the dynamical stability of HD 37124 by combining the RV data with Markov Chain Monte Carlo (MCMC) analyses to obtain ensembles of masses, semimajor axes, eccentricities, and orbital angles consistent with the RV data. These

\footnotetext{
14 This solution is of similar quality to the best-fit Newtonian solution and is dynamically stable. We consider it representative of the ensemble of good Newtonian solutions.
}

ensembles were generated without regard to dynamical stability considerations. We then imposed line of sight and relative inclination distributions on these sets of parameters. By incorporating the unknown inclination parameters with observationderived parameters, we sampled the entire phase space of orbital parameters. We subsequently ran $N$-body simulations on each element in these ensembles in order to assess each system's stability and resonant evolution. Our treatment follows that of Ford $(2005,2006)$ and Veras \& Ford $(2009,2010)$.

In particular, we calculated five Markov chains, each containing over $10^{6}$ states. Each state includes the orbital period $(P)$, velocity amplitude $(K)$, eccentricity $(e)$, argument of pericenter measured from the plane of the sky $(\omega)$, and mean anomaly at a given epoch $(u)$ for planets b, c, and d. The MCMC uses a standard Gaussian random walk proposal distribution and the Metropolis-Hastings algorithm for accepting or rejecting each proposal for all model parameters except $\cos \left(i_{\mathrm{LOS}}\right)$ and $\Omega$. Since the RV signature is only weakly dependant on these values, $\cos \left(i_{\mathrm{LOS}}\right)$ and $\Omega$ were drawn randomly for each state. This can still be considered a Markov chain, as the procedure satisfies the Markov condition, i.e., that a trial state not depend on states other than the current state, as well as the other conditions (timehomogeneous, irreducible, aperiodic) to prove that the Markov chain will (eventually) converge to the posterior distribution.

We imposed an isotropic distribution of line-of-sight inclinations $\left(i_{\mathrm{LOS}}\right)$ and a uniform sample of longitude of ascending nodes $(\Omega)$ on our MCMC-derived initial conditions. The planet masses, $m$, and semimajor axes, $a$, were obtained from each set of $(P, K, e, \omega, i, \Omega, u)$ values from relations derived with a Jacobi coordinate system (Lee \& Peale 2003). The approximate range of minimum masses obtained, in Jupiter masses, were $0.60 \lesssim m_{b} \sin i_{b}<0.72,0.40 \lesssim m_{c} \sin i_{c}<0.75$, and $0.55 \lesssim m_{d} \sin i_{d}<0.90$.

We treated both the offset between the chips and the jitter as free parameters. ${ }^{15}$ The 5 th percentile, median, and 95 th percentile offsets between the chips in our ensemble were 3.16, 3.78 , and 4.62, and the median jitter we find to be $4 \mathrm{~m} \mathrm{~s}^{-1}$.

\subsection{Coplanar, Prograde Systems}

We integrated 850 sets of initial conditions in the coplanar case with all three planets in prograde orbits by using the Bulirsch-Stoer integrator of Mercury (Chambers 1999) for $10^{7} \mathrm{yr}$ with an output interval of $10^{4} \mathrm{yr}$. We also incorporated the effects of general relativity in the code, which could have profound consequences for multiplanet system stability (Veras \& Ford 2010), although the effect is likely to be negligible in this system. We classified systems as "unstable" if, for any planet, $\left|a_{\max }-a_{\min }\right| / a_{0}>\tau$, where $a_{\max }, a_{\min }$, and $a_{0}$ represent the maximum, minimum, and initial values, respectively, of the semimajor axis, and $\tau=0.9$.

One may visualize a representative architecture of HD 37124 by comparing the semimajor axis and eccentricity ranges of all three planets. Figure 2 plots the observed eccentricity versus derived semimajor axis for all planets in the prograde coplanar state. Black dots indicate unstable systems while green squares and red crosses indicate stable systems, and red crosses indicate systems which are in a 2:1 MMR between planets $\mathrm{c}$ and $\mathrm{d}$, according to our definition below. The figure indicates: (1) a

\footnotetext{
15 We adopted a single value of jitter for all observations; in principle, the two CCDs may display differing amounts of "instrumental jitter," such as that due to insufficient modeling of the charge transfer inefficiencies. The RMS residuals to our fit for the two detectors were 3.67 and $4.11 \mathrm{~m} \mathrm{~s}^{-1}$, suggesting that our assumption of a single jitter value is valid.
} 

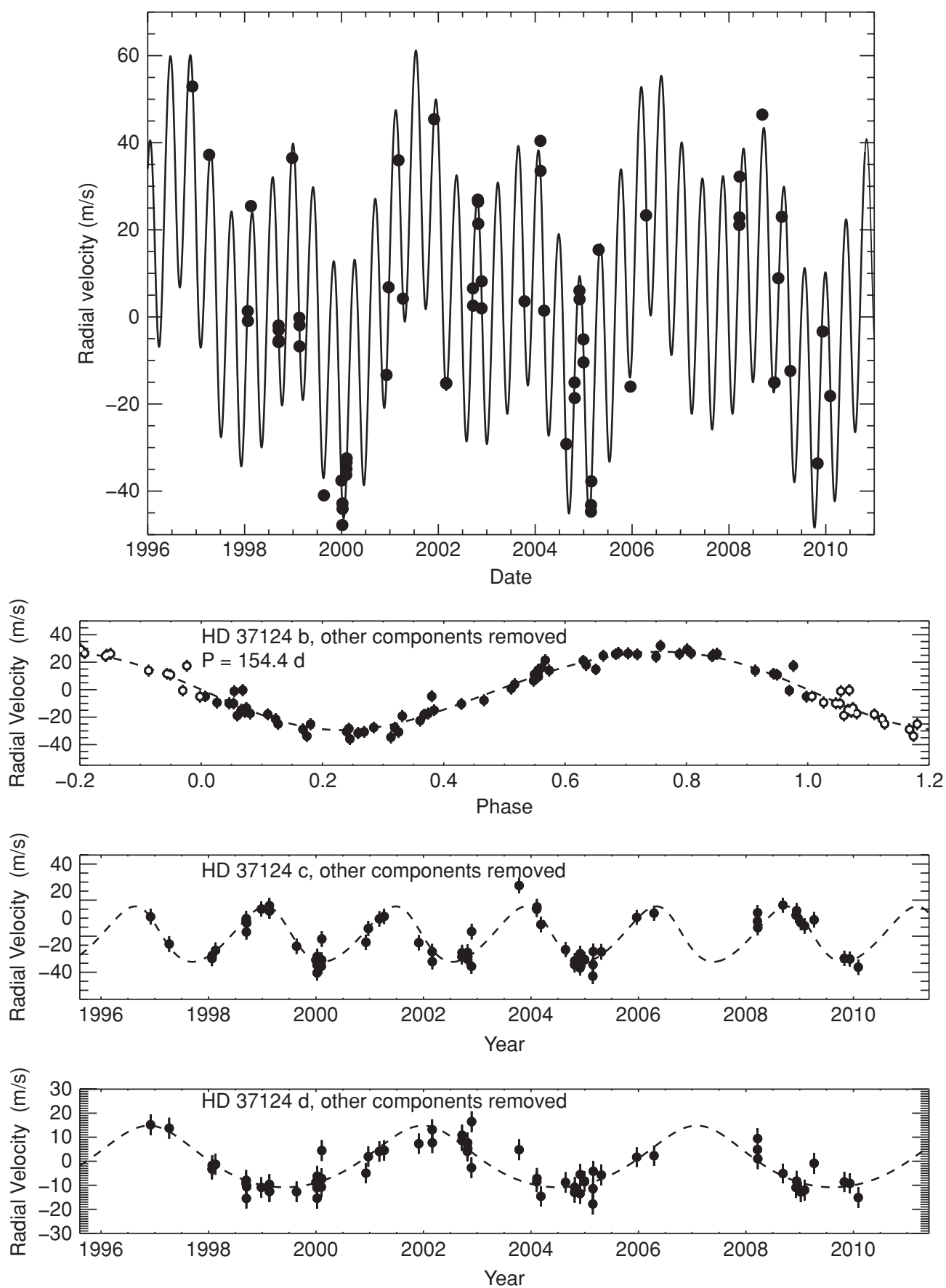

Figure 1. Radial velocity curves for the HD 37124 triple system.

closely packed system, with the inner and outer planets separated by no more than six times the innermost planet's semimajor axis, (2) a relatively circular innermost planet (with $e_{b} \lesssim 0.1$ in most cases) that is likely too far from the parent star to be classified as a "Hot Jupiter," (3) the greater the number of orbital periods sampled by $\mathrm{RV}$, the greater the constraint on the planet's likely semimajor axes and eccentricities, (4) most $(664 / 850=78 \%)$ current orbital fits predict an unstable system, (5) the majority of initial conditions which produce stable orbits contain an outer planet with a low $(<0.2)$ eccentricity, and a middle planet with a semimajor axis $>1.695 \mathrm{AU}$ and eccentricity less than about 0.2 , and (6) systems containing a 2:1 resonance occur only when $2.7 \mathrm{AU} \lesssim a_{3} \lesssim 2.8 \mathrm{AU}$. We emphasize that this approximate semimajor axis range appears to be necessary but not sufficient for resonance to occur. The figure demonstrates that other MCMC fits with outer planet periods in the resonant range are either unstable or stable but non-resonant. The architecture of these systems (as defined by, e.g., the mean longitude and longitude of pericenter) does not allow them to settle into resonance even though the outer planet period might favor resonance.

Because of finite sampling, our definition of "resonance" in this analysis comes from consideration of the RMS deviation of each resonant angle about each of $\left(0^{\circ}, 90^{\circ}, 180^{\circ}\right.$, and $\left.270^{\circ}\right)$, which includes common libration centers. We flag systems as "resonant" if at least one of these angles has RMS under $90^{\circ}$ for 


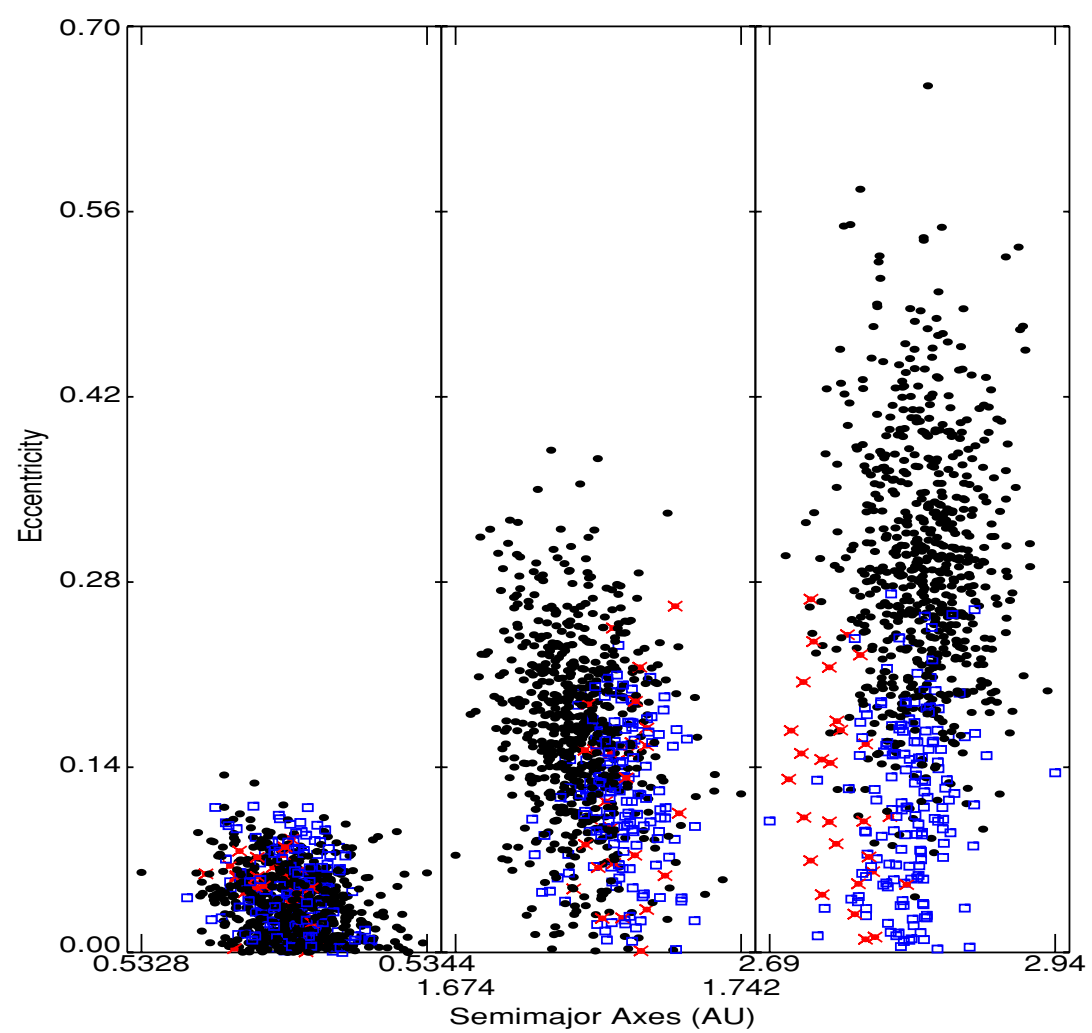

Figure 2. Representative eccentricities and semimajor axes of the planets in HD 37124. The three planets are partitioned by panels, each with a different horizontal scale. These ensembles of parameters are derived from RV observations using Markov Chain Monte Carlo (MCMC) techniques and represent the initial conditions for a subset of our numerical simulations (here the coplanar prograde simulations). Note that the semimajor axis ratio of the middle and outer planets roughly corresponds to a 2:1 period commensurability, and the inner and middle planets to a 6:1 PC. Note the changing scale on the $x$-axes in the three panels: the innermost planet is very well constrained in $a$, the outermost planet much less so. The black dots indicate unstable systems, blue squares represent non-resonant stable systems, and red x's are stable resonant systems. Note that system stability is strongly dependent on the eccentricity of the middle and outer planets, and the outer planet of resonant systems tends to harbor the smallest initial semimajor axes of the ensemble of outer planet ICs.

$10 \mathrm{Myr}$, the entire duration of our simulations. Below, we refer to this value as a "libration RMS."

HD 37124 presents a clear initial choice of angles to test for libration. As indicated by Figure 2, the semimajor axis ratio of planets $\mathrm{c}$ and $\mathrm{d}$ is suggestive of a 2:1 MMR. Therefore, we sampled the following angles for libration:

$$
\begin{aligned}
& \phi_{1} \equiv 2 \lambda_{d}-\lambda_{c}-\varpi_{c} \\
& \phi_{2} \equiv 2 \lambda_{d}-\lambda_{c}-\varpi_{d}
\end{aligned}
$$

and found that $\phi_{1}$ librates in $28 / 850=3.3 \%$ of cases, while $\phi_{2}$ librates in $9 / 850=1.1 \%$ of cases. Further, the systems for which $\phi_{2}$ is resonant are a subset of those for which $\phi_{1}$ is resonant.

If we tighten our definition of resonance to include only those systems with RMS resonant angles under $70^{\circ}$, then no $\phi_{2}$ arguments are resonant. Under this stricter definition, the $\phi_{1}$ arguments are only resonant in $14 / 850=1.6 \%$ of the cases, and if we further tighten the libration criterion to an RMS of $50^{\circ}$, then this number decreases to $4 / 850=0.5 \%$. The lowest libration RMS detected is 23.0 . All RMSs under $75^{\circ}$ were for a libration center of $0^{\circ}$. Figure 3 illustrates three examples of "resonant" systems from this, each with a different libration RMS.

We additionally sampled all three pairs of apsidal angles (the difference between two longitudes of pericenter) in the coplanar prograde state and found only two instances of libration, both at high $\left(>70^{\circ}\right)$ libration RMSs and around the "asymmetric" centers $90^{\circ}$ and $270^{\circ}$ for the inner and outer planet apsidal angle. Inspection reveals, however, that these instances of libration are more indicative of long period ( $>10 \mathrm{Myr}$ ) circulation.

Additionally, the semimajor axis ratio of planets $b$ and $c$ could indicate the presence of a 6:1 MMR. Therefore, we sampled all angles of the form $6 \lambda_{d}-\lambda_{c}-t \varpi_{c}-s \varpi_{d}$, where $t+s=5$. None of the coplanar prograde systems exhibited libration of any of the 6:1 angles between planets "b" and "c" over $10 \mathrm{Myr}$. However, preliminary sampling of these angles over intervals of $2 \mathrm{Myr}$ does occasionally exhibit libration RMSs close to $90^{\circ}$. Because the period ratios between planets $\mathrm{c}$ and $\mathrm{d}$ may skirt the 7:3 PC, we also tested the $7 \lambda_{d}-3 \lambda_{c}-t \varpi_{c}-s \varpi_{d}$ angles, where $t+s=4$, but found no instances of libration.

\subsection{Mutually Inclined Systems}

Having analyzed the coplanar prograde bin, we can now consider the case where the planets have nonzero mutual inclinations. We used rejection sampling to obtain triplets of $i_{\text {LOS }}$ values such that the system is placed into one of 144 bins according to the relative inclination between planets $b$ and $\mathrm{c}\left(i_{\text {rel, }, \mathrm{b}, \mathrm{c}}\right)$ and planets $\mathrm{c}$ and $\mathrm{d}\left(i_{\text {rel }, \mathrm{c}, \mathrm{d}}\right)$. In no two bins were the same ensemble of initial conditions used. We binned relative inclinations in intervals of $15^{\circ}$ and used stratified sampling in order to obtain a uniform number of samples per bin. We initially sampled 100 initial system states per bin. For those bins where we found more than one system to be stable, we added 200 additional ensembles of initial conditions. 

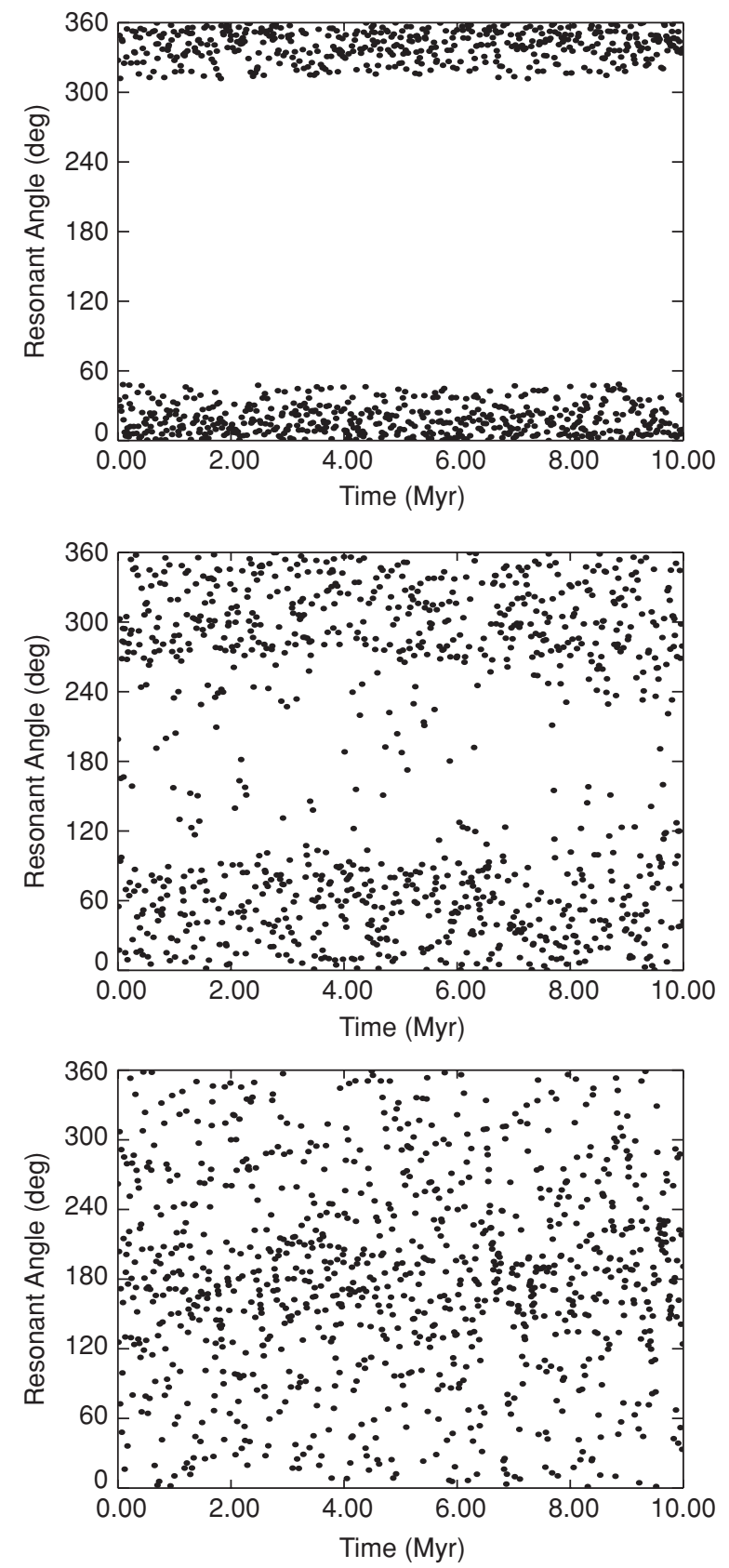

Figure 3. Three examples of systems that we find to be resonant, according to our definition requiring a libration of under $90^{\circ}$ for $10 \mathrm{Myr}$. Plotted is the time evolution of the resonant angle $2 \lambda_{d}-\lambda_{c}-\varpi_{c}$ for a system with a computed libration RMS of (upper panel) 23.0 about $0^{\circ}$, (middle panel) 70.2 about $0^{\circ}$, and (lower panel) 86.1 about $180^{\circ}$.

By considering the fraction of stable systems in the noncoplanar cases, we can obtain a broader dynamical portrait of this system. Figure 4 illustrates the fraction of stable systems in each bin overall (top panel) and with respect to all systems for which the initial $e_{d}<0.2$ (bottom panel). This cutoff was motivated by the rightmost panel in Figure 2 and could suggest a constraint on the orbital properties of the system in order to ensure that it remains stable. Figure 4 shows that the system must be roughly coplanar, with relative inclinations less than $\sim 30^{\circ}-45^{\circ}$, in order to be stable. This constraint allows various pairs of planets to harbor retrograde orbits. We also performed limited resonant testing for systems in these bins. The fraction of total systems which exhibit libration of $\phi_{1}$ and $\phi_{2}$ under $90^{\circ}$
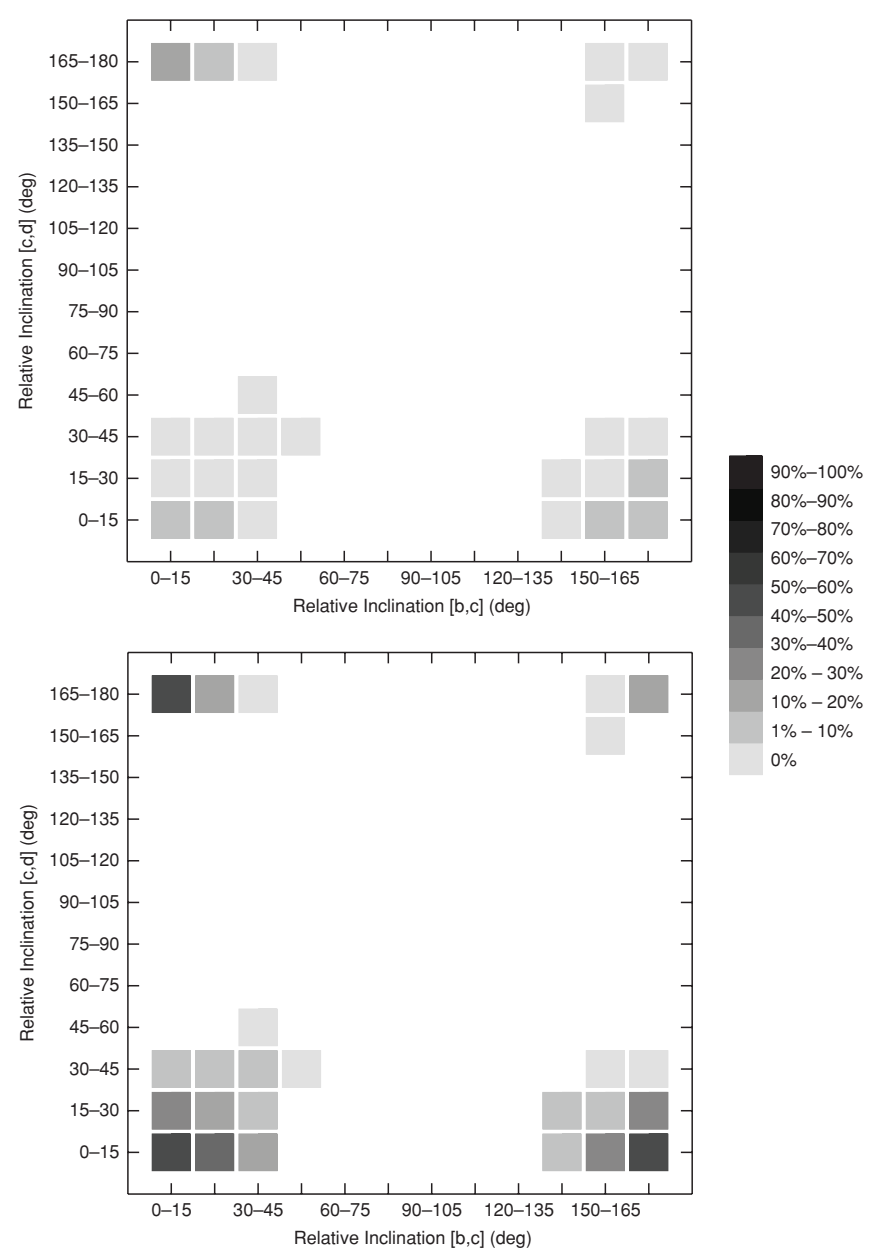

Figure 4. Two stability portraits of HD 37124. Each bin indicates the fraction of stable systems after $10 \mathrm{Myr}$ for all systems (top palette) and for systems with an initial $e_{d}<0.2$ (bottom palette). Note that nearly all non-coplanar systems are unstable (indicated by the white spaces) in both various prograde and retrograde cases. These portraits can provide a useful constraint on the viable relative inclinations in HD 37124. Note, however, that the mutual inclination of planets $\mathrm{b}$ and $\mathrm{d}$ is not represented on this plot and is only weakly constrained by the other two inclination pairs (e.g., if two pairs are mutually inclined by $30^{\circ} \mathrm{each}$, then the third pair may be mutually inclined anywhere between $0^{\circ}$ and $60^{\circ}$ ).

for $10 \mathrm{Myr}$ is given in Figure 5. 2:1 resonant systems occur, therefore, generally at the few percent level, and most likely when all planets are coplanar with prograde orbits.

\section{DISCUSSION}

As Rivera et al. (2010) showed for the GJ 876 system, even the most well-established and deepest MMRs can prove illusory if additional planets are found in the system (although in that case it appears that the resonance still present, albeit considerably shallower and more complex than previously thought). Even for truly resonant systems, a demonstration of resonance can be difficult. For instance, triple-planet systems may feature two planets with a mostly librating resonant argument that occasionally circulates due to interactions with the third planet. Near separatrix behavior (as in the case of $v$ And; Malhotra 2002; Ford et al. 2005) can also make libration and circulation essentially indistinguishable.

We note that near-resonant behavior can itself be dynamically interesting: the 5:2 near-resonance of Jupiter and Saturn (the Great Inequality) has major consequences for the dynamics of the solar system. Given the above-mentioned difficulties in 

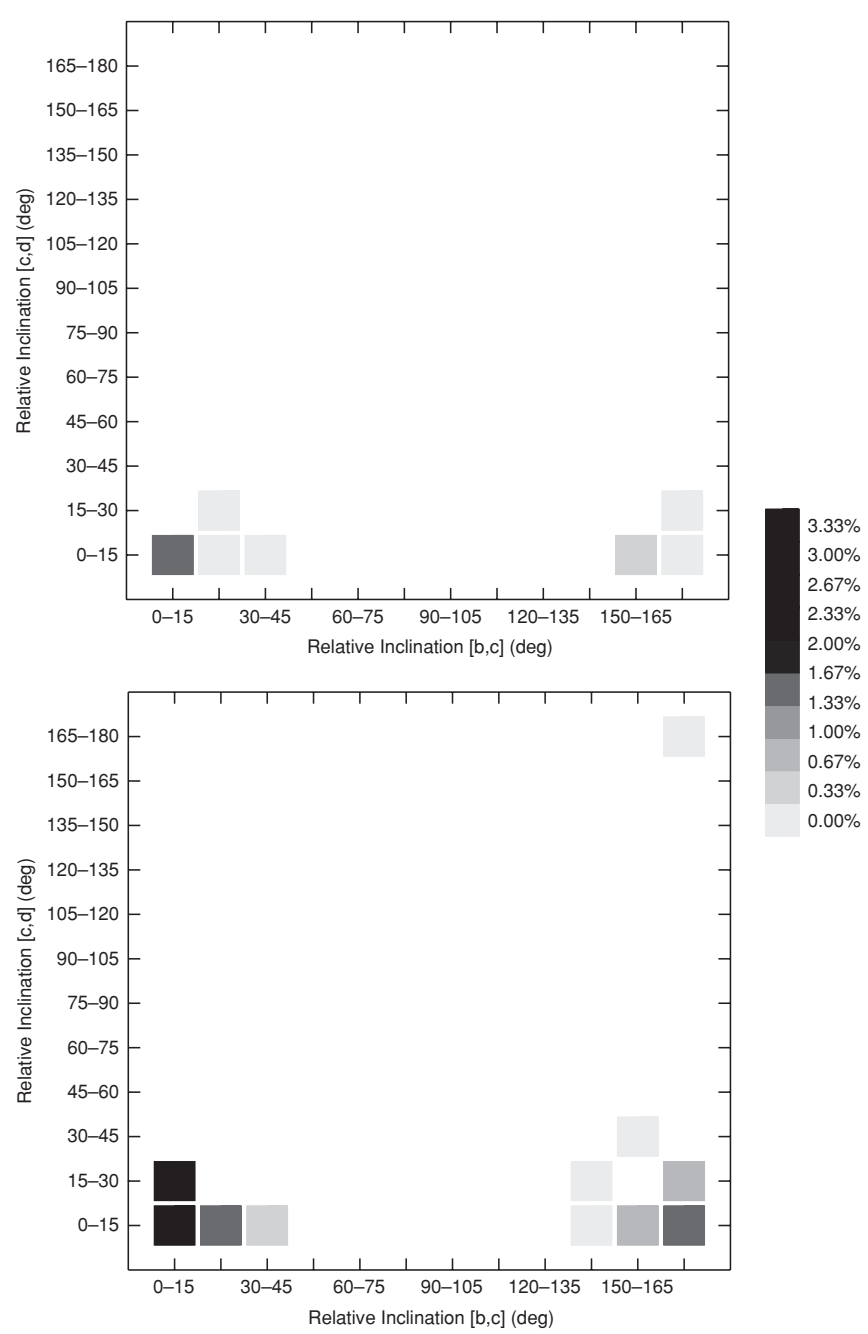

Figure 5. Two resonant portraits of HD 37124. The legend indicates the fraction of systems for which the angles $\phi_{1} \equiv 2 \lambda_{d}-\lambda_{c}-\varpi_{c}$ (upper panel) and $\phi_{1} \equiv 2 \lambda_{d}-\lambda_{c}-\varpi_{d}$ (lower panel) are resonant. We define "resonant" as the situation where the RMS deviation of an angle about a fixed value is less than $90^{\circ}$ over 10 Myr. In most cases, this deviation is between $70^{\circ}$ and $90^{\circ}$, but goes as low as $23^{\circ}$. Note that only for the near-coplanar cases are any systems resonant.

proving that a resonant argument for a given system of planets satisfies some precisely specified definition of libration given the typical uncertainties in RV measurements, we suggest that studies of resonant interactions would benefit from identifying systems that appear to be in or near resonance (apparent PCs). With that in mind, we note that in addition to HD 37124 , there are 19 other systems in the peer-reviewed ${ }^{16}$ literature with well-established apparent PCs, which we present in Table 3. This list includes all pairs of planets for which the period ratio $r$ is less than 6 and within 0.05 of an integer or half-integer (neglecting uncertainties in periods), and other exoplanetary pairs whose likely MMRs are discussed in the literature.

The fraction of known multiplanet systems exhibiting at least one apparent PC is high. Of the 43 well-determined multiplanet systems discovered by RVs around normal stars, 15 appear in Table 3 , or $35 \%$, including 9 of the 30 apparent double-planet

\footnotetext{
16 We have included the Kepler multiplanet systems, which had not yet been
} accepted for publication at the time of this writing. systems $30 \% .{ }^{17}$ To determine if this is more than would be expected simply by chance, we have performed two tests.

In the first test, we randomly drew pairs of periods from the 340 RV-discovered planets in the Exoplanet Orbit Database (EOD; Wright et al. 2011) and rejected those pairs with period ratios $r<1.3$ (corresponding to the smallest $r$ among real multiplanet systems). We counted the fraction of remaining systems with $r$ within 0.05 of an integer or half-integer $\leqslant 5$ (corresponding to the largest apparent PC in Table 3). We found that only $4 \%$ of our random pairs satisfy our apparent PC criterion, far smaller than the $30 \%$ of double-planet systems actually found in apparent PCs.

In the second test, we included the effects of triple and highermultiple systems by randomly assigning periods from the EOD to all planets in real multiple systems (again subject to the constraint that no pair of planets in the system have $r<1.3$ ). We found $16 \%$ of these artificial systems passed our apparent PC criterion, reflecting the higher number of planet pairs available to test per star compared to our first test. Despite this inflation, the actual value of $33 \%$ among all multiplanet systems is still significantly higher. ${ }^{18}$ These results underscore that the orbital periods of the population of planets known to be in multiplanet systems are inconsistent with the apparently singleton sample (Wright et al. 2009a).

This apparently high percentage of known multiplanet systems with an apparent PC might favor particular formation mechanisms. Planet-planet scattering, planetesimal disk migration, and gas disk migration have all been shown to produce systems with at least one pair of planets that are not only commensurate in period but also resonant. Raymond et al. (2008) found that planet-planet scattering produced MMRs in roughly $5 \%$ of the systems that they simulated, and Raymond et al. (2010) discovered that between $50 \%$ and $80 \%$ of systems undergoing planetesimal disk migration yielded resonant capture. Convergent gas disk migration, the thrust of the numerous papers cited in Section 1, can occur with near $100 \%$ efficiency for certain initial planetary and disk parameters. As observed by Thommes \& Lissauer (2003) and Libert \& Tsiganis (2009), the inclination may be excited as well as the eccentricity in many resonant cases. If a resonance exists in HD 37124, it could have been produced by any of these methods. If the RMS libration of such a resonance is representative of the bottom panel of Figure 3 and has a value that approaches $90^{\circ}$, then planet-planet scattering is a likely origin of this resonance. Alternatively, disk or gas migration would likely produce a system that is "deeper" in resonance, with a smaller variation in resonant angle, similar to the top panel of Figure 3. Resonant librating angles need not involve the eccentricities and pericenters, but instead the inclinations and longitudes of ascending nodes, similar to the $4: 2$ Mimas-Tethys resonance in the Saturnian system (Champenois \& Vienne 1999a, 1999b).

\footnotetext{
17 We have excluded in this statistic the Kepler systems, the pulsar system, the microlensing system, planets from direct imaging, and the solar system. We acknowledge that a more rigorous statistic would be valuable, but note that it would need to address some strong detectability and selection effects regarding planets in multiple planet systems and to assess these detection thresholds across multiple, heterogeneous surveys. To give just one example, we note that in addition to an RV survey's decreasing sensitivity to planets in longer periods, it can be difficult to detect an interior planet in a 2:1 resonance due to approximate degeneracy with eccentricity in a single-planet model (e.g., Anglada-Escudé et al. 2010; Moorhead \& Ford 2010). Such an analysis is beyond the scope of this manuscript.

18 We suspect that the reason the observed value is not similarly inflated with respect to double-planet systems is that our randomization did not include the requirement of dynamical stability, as real systems implicitly do.
} 
Table 3

Apparent Period Commensurabilities in Well-characterized Multiplanet Systems

\begin{tabular}{|c|c|c|c|}
\hline System & Components & Period Ratio & References \\
\hline GJ 876 & $e, c, b$ & $4: 2: 1$ & Marcy et al. (2001); Rivera et al. (2010) \\
\hline HD 82943 & $b, c$ & $2: 1$ & Mayor et al. (2004); Ji et al. (2003); Lee et al. (2006) \\
\hline HD 37124 & $c, d$ & $2: 1$ & Vogt et al. (2005); this work \\
\hline HD 128311 & $c, b$ & $2: 1$ & Sándor et al. (2007); Vogt et al. (2005); others \\
\hline HD 73526 & $c, b$ & $2: 1$ & Tinney et al. (2006); Sándor et al. (2007) \\
\hline$\mu$ Ara & $b, e$ & $2: 1$ & Pepe et al. (2007); Goździewski et al. (2007) \\
\hline KOI $152^{\mathrm{a}}$ & 2,3 & $2: 1$ & Steffen et al. (2010) \\
\hline KOI $877^{\mathrm{a}}$ & 2,1 & $2: 1$ & Steffen et al. (2010) \\
\hline $24 \mathrm{Sex}$ & $c, b$ & $2: 1$ & Johnson et al. (2011) \\
\hline Kepler-9 & $c, b$ & $2: 1$ & Holman et al. (2010) \\
\hline PSR B $1257+12$ & $B, C$ & $3: 2$ & Wolszczan \& Frail (1992); Malhotra et al. (1992); Konacki et al. (1999) \\
\hline HD 45364 & $c, b$ & $3: 2$ & Rein et al. (2010); Correia et al. (2009) \\
\hline HD 200964 & $c, b$ & $4: 3$ & Johnson et al. (2011) \\
\hline $55 \mathrm{Cnc}$ & $c, b$ & $3: 1$ & Fischer et al. (2008); Zhou et al. (2008) \\
\hline HD 10180 & $d, e$ & $3: 1$ & Lovis et al. (2010) \\
\hline HD 60532 & $c, b$ & $3: 1$ & Desort et al. (2008, 2009); Laskar \& Correia (2009) \\
\hline HD 108874 & $c, b$ & $4: 1$ & Vogt et al. (2005); Goździewski et al. (2006) \\
\hline Solar & $\hbar, 4$ & $5: 2$ & \\
\hline HD 10180 & $e, f$ & $5: 2$ & Lovis et al. (2010) \\
\hline KOI $896^{\mathrm{a}}$ & 1,2 & $5: 2$ & Steffen et al. (2010) \\
\hline HD 202206 & $c, b$ & $5: 1$ & Correia et al. (2005); Goździewski et al. (2006) \\
\hline
\end{tabular}

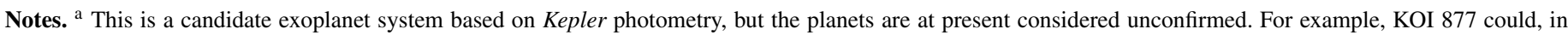
principle, be a blend of two stars, each hosting one transiting planet in a coincidental apparent PC.

\section{CONCLUSIONS}

We have resolved the period ambiguity of HD $37124 d$ from Vogt et al. (2005) and find that HD $37124 c$ and $d$ are in an apparent 2:1 PC. Our numerical integrations show that both resonant and non-resonant configurations are consistent with the RV data and that stability requires a nearly circular orbit $(e<0.3)$ for the $d$ component. Our stability analysis shows that the system must be nearly coplanar and that the three planets have identical minimum masses within the errors (of 3\%-10\%).

We show that roughly one in three well-characterized multiplanet systems shows an apparent PC, which is more than a naïve estimate based on randomly drawing periods from the known exoplanet population would suggest. This offers evidence for some particular proposed scattering and migration mechanisms, and we suggest that the statistics of multiplanet systems may now be sufficiently robust to provide a test and comparison of models of exoplanetary dynamical evolution.

We thank the referee, Daniel Fabrycky, for his constructive and thorough review, which significantly improved this manuscript.

J.T.W. received support from NASA Origins of Solar Systems grant NNX10AI52G. D.V. and E.B.F. were partially supported by NASA Origins of Solar Systems grant NNX09AB35G. A.W.H. gratefully acknowledges support from a Townes Postdoctoral Fellowship at the UC Berkeley Space Sciences Laboratory.

This work was partially supported by funding from the Center for Exoplanets and Habitable Worlds, which is supported by the Pennsylvania State University, the Eberly College of Science, and the Pennsylvania Space Grant Consortium.

The work herein is based on observations obtained at the W. M. Keck Observatory, which is operated jointly by the University of California and the California Institute of Technology. The Keck Observatory was made possible by the generous financial support of the W. M. Keck Foundation. We recognize and acknowledge the very significant cultural role and reverence that the summit of Mauna Kea has always had within the indigenous Hawaiian community. We are most fortunate to have the opportunity to conduct observations from this mountain.

The authors acknowledge the University of Florida HighPerformance Computing Center for providing computational resources and support that have contributed to the results reported within this paper.

This research has made use of NASA's Astrophysics Data System and the Exoplanet Orbit Database at exoplanets.org.

Facility: Keck:I

\section{REFERENCES}

Anglada-Escudé, G., López-Morales, M., \& Chambers, J. E. 2010, ApJ, 709, 168

Batalha, N. M., et al. 2011, ApJ, 729, 27

Beaugé, C., Michtchenko, T. A., \& Ferraz-Mello, S. 2006, MNRAS, 365, 1160

Butler, R. P., Marcy, G. W., Fischer, D. A., Brown, T. M., Contos, A. R., Korzennik, S. G., Nisenson, P., \& Noyes, R. W. 1999, ApJ, 526, 916

Butler, R. P., Marcy, G. W., Vogt, S. S., Fischer, D. A., Henry, G. W., Laughlin, G., \& Wright, J. T. 2003, ApJ, 582, 455

Butler, R. P., Marcy, G. W., Williams, E., McCarthy, C., Dosanjh, P., \& Vogt, S. S. 1996, PASP, 108, 500

Butler, R. P., et al. 2006, ApJ, 646, 505

Chambers, J. E. 1999, MNRAS, 304, 793

Champenois, S., \& Vienne, A. 1999a, Celest. Mech. Dyn. Astron., 74, 111

Champenois, S., \& Vienne, A. 1999b, Icarus, 140, 106

Chiang, E. I., \& Jordan, A. B. 2002, AJ, 124, 3430

Correia, A. C. M., Udry, S., Mayor, M., Laskar, J., Naef, D., Pepe, F., Queloz, D., \& Santos, N. C. 2005, A\&A, 440, 751

Correia, A. C. M., et al. 2009, A\&A, 496, 521

Desort, M., Lagrange, A.-M., Galland, F., Beust, H., Udry, S., Mayor, M., \& Lo Curto, G. 2008, A\&A, 491, 883

Desort, M., Lagrange, A., Galland, F., Beust, H., Udry, S., Mayor, M., \& Lo Curto, G. 2009, A\&A, 499, 623

Fischer, D. A., et al. 2008, ApJ, 675, 790

Ford, E. B. 2005, AJ, 129, 1706

Ford, E. B. 2006, in ASP Conf. Ser. 352, New Horizons in Astronomy: Frank N. Bash Symposium, ed. S. J. Kannappan et al. (San Francisco, CA: ASP), 15 
Ford, E. B., Lystad, V., \& Rasio, F. A. 2005, Nature, 434, 873

French, R. G., McGhee, C. A., Dones, L., \& Lissauer, J. J. 2003, Icarus, 162 , 143

Gaudi, B. S., et al. 2008, Science, 319, 927

Goldreich, P. 1965, MNRAS, 130, 159

Gomes, R., Levison, H. F., Tsiganis, K., \& Morbidelli, A. 2005, Nature, 435, 466

Goździewski, K. 2003, A\&A, 398, 315

Goździewski, K., Breiter, S., \& Borczyk, W. 2008, MNRAS, 383, 989

Goździewski, K., Konacki, M., \& Maciejewski, A. J. 2006, ApJ, 645, 688

Goździewski, K., Maciejewski, A. J., \& Migaszewski, C. 2007, ApJ, 657, 546

Gregory, P. C., \& Fischer, D. A. 2010, MNRAS, 403, 731

Holman, M. J., et al. 2010, Science, 330, 51

Howard, A. W., et al. 2009, ApJ, 696, 75

Howard, A. W., et al. 2010, ApJ, 721, 1467

Ji, J., Liu, L., Kinoshita, H., Zhou, J., Nakai, H., \& Li, G. 2003, ApJ, 591, L57

Johnson, J. A., et al. 2011, AJ, 141, 16

Kley, W., Peitz, J., \& Bryden, G. 2004, A\&A, 414, 735

Konacki, M., Maciejewski, A. J., \& Wolszczan, A. 1999, ApJ, 513, 471

Laskar, J., \& Correia, A. C. M. 2009, A\&A, 496, L5

Lee, M. H., Butler, R. P., Fischer, D. A., Marcy, G. W., \& Vogt, S. S. 2006, ApJ, 641,1178

Lee, M. H., \& Peale, S. J. 2003, ApJ, 592, 1201

Libert, A., \& Tsiganis, K. 2009, MNRAS, 400, 1373

Lovis, C., et al. 2010, arXiv:1011.4994

Malhotra, R. 2002, ApJ, 575, L33

Malhotra, R., Black, D., Eck, A., \& Jackson, A. 1992, Nature, 356, 583

Marcy, G. W., Butler, R. P., Fischer, D., Vogt, S. S., Lissauer, J. J., \& Rivera, E. J. 2001, ApJ, 556, 296

Marcy, G. W., Butler, R. P., Vogt, S. S., Fischer, D. A., Henry, G. W., Laughlin, G., Wright, J. T., \& Johnson, J. A. 2005, ApJ, 619, 570

Mayor, M., Udry, S., Naef, D., Pepe, F., Queloz, D., Santos, N. C., \& Burnet, M. 2004, A\&A, 415, 391

Mayor, M., et al. 2009, A\&A, 507, 487

Moorhead, A., \& Ford, E. 2010, in EAS Publ. Ser. 42, Extrasolar Planets in Multi-Body Systems: Theory and Observations, ed. K. Gożdziewski, A. Niedzielski, \& J. Schneider, 161

Morbidelli, A., Levison, H. F., Tsiganis, K., \& Gomes, R. 2005, Nature, 435, 462

Murray-Clay, R. A., \& Chiang, E. I. 2005, ApJ, 619, 623

Nagasawa, M., Lin, D. N. C., \& Thommes, E. 2005, ApJ, 635, 578

Papaloizou, J. C. B., \& Szuszkiewicz, E. 2005, MNRAS, 363, 153

Papaloizou, J. C. B., \& Terquem, C. 2010, MNRAS, 405, 573

Pepe, F., et al. 2007, A\&A, 462, 769

Pierens, A., \& Nelson, R. P. 2008, A\&A, 482, 333
Podlewska, E., \& Szuszkiewicz, E. 2008, MNRAS, 386, 1347

Podlewska, E., \& Szuszkiewicz, E. 2009, MNRAS, 397, 1995

Raymond, S. N., Armitage, P. J., \& Gorelick, N. 2010, ApJ, 711, 772

Raymond, S. N., Barnes, R., Armitage, P. J., \& Gorelick, N. 2008, ApJ, 687, $\mathrm{L} 107$

Rein, H., \& Papaloizou, J. C. B. 2009, A\&A, 497, 595

Rein, H., Papaloizou, J. C. B., \& Kley, W. 2010, A\&A, 510, A4

Rivera, E. J., Laughlin, G., Butler, R. P., Vogt, S. S., Haghighipour, N., \& Meschiari, S. 2010, ApJ, 719, 890

Rivera, E. J., et al. 2005, ApJ, 634, 625

Sándor, Z., Kley, W., \& Klagyivik, P. 2007, A\&A, 472, 981

Santos, N. C., et al. 2004, A\&A, 426, L19

Steffen, J. H., et al. 2010, ApJ, 725, 1226

Terquem, C., \& Papaloizou, J. C. B. 2007, ApJ, 654, 1110

Thommes, E. W., \& Lissauer, J. J. 2003, ApJ, 597, 566

Tinney, C. G., Butler, R. P., Marcy, G. W., Jones, H. R. A., Laughlin, G., Carter, B. D., Bailey, J. A., \& O'Toole, S. 2006, ApJ, 647, 594

Tsiganis, K., Gomes, R., Morbidelli, A., \& Levison, H. F. 2005a, Nature, 435, 459

Tsiganis, K., Varvoglis, H., \& Dvorak, R. 2005b, Celest. Mech. Dyn. Astron., 92, 71

Tsiganis, K., Varvoglis, H., \& Hadjidemetriou, J. D. 2002, Icarus, 159, 284

Valenti, J. A., \& Fischer, D. A. 2005, ApJS, 159, 141

Veras, D., \& Ford, E. B. 2009, ApJ, 690, L1

Veras, D., \& Ford, E. B. 2010, ApJ, 715, 803

Vogt, S. S., Butler, R. P., Marcy, G. W., Fischer, D. A., Henry, G. W., Laughlin, G., Wright, J. T., \& Johnson, J. A. 2005, ApJ, 632, 638

Vogt, S. S., Marcy, G. W., Butler, R. P., \& Apps, K. 2000, ApJ, 536, 902

Vogt, S. S., et al. 1994, Proc. SPIE, 2198, 362

Wolszczan, A. 1994, Science, 264, 538

Wolszczan, A., \& Frail, D. A. 1992, Nature, 355, 145

Wright, J. T. 2005, PASP, 117, 657

Wright, J. T. 2010, in EAS Publ. Ser. 42, Extrasolar Planets in Multi-Body Systems: Theory and Observations, ed. K. Gożdziewski, A. Niedzielski, \& J. Schneider, 3

Wright, J. T., \& Howard, A. W. 2009, ApJS, 182, 205

Wright, J. T., Upadhyay, S., Marcy, G. W., Fischer, D. A., Ford, E. B., \& Johnson, J. A. 2009a, ApJ, 693, 1084

Wright, J. T., et al. 2007, ApJ, 657, 533

Wright, J. T., et al. 2009b, ApJ, 699, L97

Wright, J. T., et al. 2011, PASP, in press (arXiv:1012.5676)

Zhang, H., \& Zhou, J. 2010a, ApJ, 714, 532

Zhang, H., \& Zhou, J. 2010b, ApJ, 719, 671

Zhou, L., Ferraz-Mello, S., \& Sun, Y. 2008, in IAU Symp. 249, Exoplanets: Detection, Formation and Dynamics, ed. Y.-S. Sun, S. Ferraz-Mello, \& J.-L. Zhou (Cambridge: Cambridge Univ. Press), 485 